\title{
A case series on drug induced hyponatremia: uncommon adverse effect of commonly used drugs
}

\author{
Harsh M. Joshi ${ }^{1}$, Sapna Gupta ${ }^{2 *}$, Supriya Malhotra ${ }^{1}$, Pankaj Patel ${ }^{3}$
}

${ }^{1}$ Department of Pharmacology, N.H.L Municipal Medical College, Ahmedabad, Gujarat, India

${ }^{2}$ Department of Emergency Medicine, V.S. Hospital, Ahmedabad, Gujarat, India ${ }^{3}$ Dean, N.H.L Municipal Medical College, Ahmedabad, Gujarat, India

Received: 08 April 2018 Accepted: 01 May 2018

\section{*Correspondence to: \\ Dr. Sapna Gupta, \\ Email: sapna_gupta76@ \\ yahoo.com}

Copyright: (C) the author(s), publisher and licensee Medip Academy. This is an openaccess article distributed under the terms of the Creative Commons Attribution NonCommercial License, which permits unrestricted noncommercial use, distribution, and reproduction in any medium, provided the original work is properly cited.

\begin{abstract}
Older patients suffering from depression and psychosis have markedly increased since last decade. So, has the use of antidepressants and antipsychotics. The prevalence of hyponatremia due to these drugs is common in general as well as psychiatric practice. It may also lead to life threatening morbidity and mortality. Loss of renal function, polypharmacy, dementia and other conditions of advanced age can either exacerbate the severity of hyponatremia or mask its onset. In this case series, total four cases were reported of hyponatremia and drugs causing it were escitalopram, quetiapine, tianeptine and oxcarbazepine. Due to polypharmacy, a chance of hyponatremia was more in these patients. Patients received infusion of hypertonic saline with salt added diet to treat hyponatremia. Symptoms of hyponatremia were improved after the treatment. In all four cases, WHO and Naranjo's causality assessment revealed 'possible' causal relationship with the prescribed drug. Prescribers should be aware of such adverse effect due to these drugs.
\end{abstract}

Keywords: Escitalopram, Hyponatremia, Oxcarbazepine, Polypharmacy, Quetiapine, Tianeptine

\section{INTRODUCTION}

Hyponatremia, usually defined as a serum concentration of $\mathrm{Na}^{+}$below $135 \mathrm{mEq} / \mathrm{L}$, is the most prevalent electrolyte aberrance encountered in hospitalized patients in both general medical and psychiatric institutions. ${ }^{1}$ Signs and symptoms mainly are neuropsychiatric and gastrointestinal.

The prevalence of hyponatremia has been estimated as $15 \%$ and $11 \%$ in general and psychiatric hospital settings, respectively. Hyponatremia has been linked with increased health care resource use and costs. ${ }^{2}$ It can cause significant morbidity and by way of an osmotic demyelinating encephalopathy, may lead to serious consequences, such as lethargy, confusion, coma, and occasionally death.

Among psychiatric patients, hyponatremia is believed to be frequently underdiagnosed because clinical signs may mimic psychopathological symptoms of the underlying condition. Physiological serum $\left[\mathrm{Na}^{+}\right]$is maintained within tight limits through a complex homeostatic system involving hypothalamic osmoreceptors and peripheral 
baroreceptors regulating water intake and urine output via changes in thirst and the neurohypophyseal secretion of the antidiuretic hormone arginine-vasopressin (AVP). ${ }^{3}$ Case reports, case control, and population-based studies have implicated antidepressants, such as tricyclic antidepressants (TCAs), selective serotonin reuptake inhibitors (SSRIs), selective serotonin and norepinephrine reuptake inhibitors (SNRIs), and certain anticonvulsants mainly carbamazepine and oxcarbazepine in hyponatremia of this origin. ${ }^{4}$

Age and female sex are established major risk factors for treatment-emergent hyponatremia. Moreover, in psychiatry, special patient populations are at particular risk to develop this type of electrolyte disturbance. These include mainly schizophrenia patients with polydipsia or others with compulsive drinking behavior and individuals with anorexia-related surreptitious water loading. ${ }^{5}$

Occurrence of polydipsia and polyuria in schizophrenia were reported already in the early part of the 20th century, before specific psychiatric drug treatment became available. Despite the apparent clinical significance of the issue, our knowledge on prevalence, risk factors, and drugs involved in treatment-related hyponatremia is mainly confined to antidepressants, in particular SSRIs and venlafaxine. $^{6}$

Elderly patients are particularly at risk for hyponatremia in general, since many are taking diuretics for hypertension, while some studies have shown to increase the risk of SSRI-associated hyponatremia. Loss of renal function, polypharmacy, dementia, and other conditions of advanced age can either exacerbate the severity of hyponatremia or mask its onset. ${ }^{7}$ Reporting of this case series as an attempt by the authors to highlight the nature of the problem as all these patients presented to the emergency medicine department. Further, this case series also substantiates drug induced hyponatremia to be common in patients. Hyponatremia can also result from drug interactions occurring from concomitant medications and physicians should be careful in this regard.

\section{CASE REPORT}

\section{Case 1}

A 60 year old female admitted to a tertiary care hospital in emergency ward with complaints of drowsiness and altered sensorium since one day and an episode of convulsion one day before. On examination her temperature was normal, heart rate was decreased (60/minute) while blood pressure was higher $(140 / 90 \mathrm{~mm}$ of $\mathrm{Hg}$ ). She was deeply comatose and did not responded to verbal commands. She was promptly intubated to secure the airway and was given ventilator support. On investigations, her haemoglobin was $8.45 \mathrm{gm} \%$, total count was $18,200 /$ cumm, platelet count was $64000 /$ cumm, renal function test was normal while serum sodium was $113 \mathrm{mg} / \mathrm{L}$ (hyponatremia). Her random blood sugar was
$204 \mathrm{mg} \%, \mathrm{pH}=7.40$ while bicarbonate level was within normal range. On asking the history, she was a known case of psychiatric illness since 9 years and was on tablet nortriptyline $(25 \mathrm{mg})$ one tablet twice a day, tablet escitalopram $(10 \mathrm{mg})$ one tablet before bed time and tablet nitrazepam (5mg) one tablet before bed time. On admission her psychiatric reference was done. On examination, she had fluctuating levels of consciousness and was pulling of intravenous lines of her own. She was diagnosed to have delirium and was prescribed tablet haloperidol (1.5mg) twice a day. Her mini-mental state scale score was 6 while Revised Delirium Rating Scale (DRS-98) score was 20. She was on ventilator for four days. Hyponatremia gradually recovered over next 10 days. Her sensorium was improved. On recovery she had complaints of forgetfulness, sleep disturbance and inability to identify family members. Her mini-mental state examination (MMSE) improved to 28 and her DRS$\mathrm{R}$ score was 10 . After 10 days, serum sodium levels were repeated which was within the normal range (135meq/l). WHO and naranjo's causality assessment revealed 'possible' causal relationship as escitalopram induced hyponatremia.

\section{Case 2}

A 40 year old female admitted to a tertiary care teaching hospital with complaints of incontinence of urine and stools since three days, yellow discoloration of skin since five days, drowsiness and irrelevant talking since 15 days. On examination, her temperature was normal, heart rate was 90 per minute, respiratory rate was normal and blood pressure was $114 / 70 \mathrm{~mm}$ of $\mathrm{Hg}$. On central nervous system examination, she could move all four limbs, no neck rigidity but irritability and spasticity were present. Muscle power was 3 out of 5. On taking the history, she was given treatment for psychiatric illness since 20 years. Presently she was on tablet quetiapine $(50 \mathrm{mg}$ ) before bed time, tablet trifluoperzine and haloperidol twice a day, tablet amisulpride $(50 \mathrm{mg})$ twice a day and tablet lorazepam (2mg) when needed since one month. She was a known case of polycystic kidneys with SLE (systemic lupus erythematosis). Her serum sodium on admission was $108 \mathrm{meq} / \mathrm{L}$. She was given infusion of hypertonic saline with salt added diet and sensorium improved over next 45 days. WHO and naranjo's causality assessment revealed 'possible' causal relationship with the drugs.

\section{Case 3}

A 58 year old female from low socioeconomic status and poor social support came to tertiary care hospital with excessive weakness, lethargy, muscle pain, cramps, unsteady gait, disorientation, urinary retention of urine and the patient was admitted to the emergency ward. Emergency treatment starts and on examination revealed pallor over tongue, mild tachycardia (80/minute) while blood pressure $(130 / 80 \mathrm{~mm}$ of $\mathrm{Hg})$, respiratory rate and temperature was normal. His blood investigations revealed Serum sodium was $116 \mathrm{mmol} / \mathrm{l}$, Serum potassium was 
$3.2 \mathrm{mmol} / \mathrm{l}$ and total leucocyte count was $12,200 / \mathrm{cumm}$. Other investigations were within normal range. On taking history, she was a known case of hypertension since nine years and was on Tablet enalapril $5 \mathrm{mg}$ per day. She was having psychiatric illness two years before and diagnosed to have as severe depression as per ICD-10 (DCR) and was on tablet tianeptine $(12.5 \mathrm{mg})$ one tablet twice a day, tablet clonazepam $(0.5 \mathrm{mg})$ one tablet twice a day and tablet escitalopram $(5 \mathrm{mg})$ one tablet before bed time. Before four weeks her depression was improved, sleep pattern was restored and symptoms abated. So she continued treatment with the same dose for longer period of time. Suddenly she developed mentioned symptoms and need to be admitted. Looking on investigations, she had hyponatremia and was treated with hypertonic saline and large salt diet. She recovered in two days and shifted to recovery ward. Her symptoms and general condition improved over the next two days and serum sodium level reached $140 \mathrm{mmol} / \mathrm{l}$. She was discharged on fourth day. The patient was educated about the risk of hyponatremia, fluid intake habits, importance of diet plan and early warning signs about hyponatremia. By the WHO-UMC scale (WHO) and Naranjo algorithm, "possible" causal relation between the drug and adverse event was established.

\section{Case 4}

A 66 year old male admitted in a tertiary care hospital with difficulty in walking, drowsiness, slurred speech since one day, problem in gait since two day and generalized weakness since one week. On examination he was found to have disorientation to place, time and person, impairment of memory, poor attention and concentration is decreased. Physical examination was done, and it did not reveal any signs of dehydration or hypervolaemia. His pulse rate was $82 /$ minute and blood pressure were $110 / 70 \mathrm{~mm}$ of $\mathrm{Hg}$. On asking the history, there is no evidence of head injury, taking over the counter medications, restriction of diet, drug or alcohol abuse and excessive intake of water. His computerized tomography of brain was normal. Liver function test, random blood sugar levels, serum electrolytes, serum urea and creatinine was within normal range. His serum sodium level was $116 \mathrm{meq} / \mathrm{l}$ showed hyponatremia. He was a known case of bipolar depression since five years and since last one month he was prescribed tablet oxcarbazepine (300mg) once a day, tablet oxcarbazepine $(600 \mathrm{mg})$ before bed time, tablet lorazepam (2mg) before bed time and tablet olanzapine $(10 \mathrm{mg})$ before bed time. Treatment was stated in the form of intravenous sodium chloride (3\%) and high salt diet. The patient improved gradually over 5 days.

On recovery his psychiatric evaluation was done which showed excessive talkativeness and manic behavior. He has slurred speech and hypersalivation. He was prescribed tab olanzapine $(5 \mathrm{mg}$ ) before bed time and tablet quetiapine (25mg) half tablet in the morning and evening while one tablet before bed time. He recovered completely over next ten days after admission and on investigations his serum sodium level revealed $138 \mathrm{mmol} / \mathrm{l}$ (within normal range).
By the WHO-UMC scale (WHO) and Naranjo algorithm, "possible" causal relation between the drug and adverse event was established.

\section{DISCUSSION}

Hyponatremia by drugs prescribed for psychiatric illnesses is a complex and multifactorial phenomenon which can present as an ADR (adverse drug reaction) as well as in frequent correlation to varieties of co-morbities. It can occur due to polydipsia as a symptom in patients of psychosis, a common and unreported symptom by the patient which can worsen hyponatremia. In this case series, three out of four cases of the hyponatremia was more than 50 years which shows correlation of age with hyponatremia. These findings are similar with the current studies in which age is one of the most predictive factor of hyponatremia may be due to multiple reasons. ${ }^{8}$

As we know, older patients have various co-morbidities and results in polypharmacy including some drugs having a known adverse effect as hyponatremia such as thiazide diuretics, ACEI etc. As increasing age and kidney functions are altered, causes decreased excretion of drugs and ultimately more incidence of hyponatremia. ${ }^{9}$ The majority of hyponatremia cases in the elderly and most of the cases occurring in psychiatric patients have been attributed to SIADH.

Hyponatremia due to drugs may occur because of the following reasons. The first reason being that there is no proper physiological stimuli but increased secretion of hypothalamic production and hypophyseal release of AVP (arginine-vasopressin) secondary to SIADH (like antipsychotic, antidepressants) the second explanation especially for iminostilbene group of anticonvulsant drugs causing hyponatremia is via potentiating the endogenous AVP release in the kidney at the level of renal tubule. Another plausible mechanism states decrease threshold of AVP secretion by resetting the osmostat. Carbamazepine and oxcarbazepine causes hyponatremia via above three mechanisms. ${ }^{10}$

Hyponatremia due to antidepressants like TCAs, SNRIs, and SSRIs have one another mechanism that serotonin (5HT) can cause release of AVP from neurohypophysis. When antidepressants like SSRI combined with ACEI the risk of hyponatremia is increased.

Occurrence of hyponatremia during treatment with SSRI ranges between 0.5 and $25 \% .{ }^{11}$ The commonest serotoninreuptake inhibitors (SSRIs), causing hyponatremia is reported to be due to citalopram but some reports also convincing for hyponatremia associated with escitalopram. Occurrence of hyponatremia is much more if SSRI is taken along with other antidepressants in combination. ${ }^{12}$ During first week of starting the therapy, risk of hyponatremia is highest, and it is more in women than in men of geriatric population. ${ }^{12}$ In the first case, tab escitalopram $(10 \mathrm{mg})$ was continued since 9 years and 
onset of hyponatremia is seen after long time of taking the drug. Tablet Nortriptyline and tablet nitrazepam are less likely to cause hyponatremia. While in third case, escitalopram $(5 \mathrm{mg})$ was prescribed with tablet tianeptine and tablet clonazepam. But she was on ACEI for hypertension which contributes to hyponatremia. In this case, by WHO and Naranjo criteria, we found 'possible' causal relationship with the drugs which indicated that escitalopram possibly caused the hyponatremia.

The syndrome of inappropriate antidiuretic hormone (SIADH) secretion is characterized by a low serum sodium concentration ( $<135 \mathrm{mmol}$ per liter), urinary osmolality exceeding 200mOsm per kilogram, a urinary sodium concentration exceeding $20 \mathrm{mmol}$ per liter, and serum osmolality of less than $280 \mathrm{mOsm}$ per kilogram. ${ }^{13}$ So Senantiomer of citalopram, escitalopram is can be the causative drug for the hyponatremia.

Causes of SIADH are 1) CNS diseases like stroke, infection, trauma, hemorrhage and psychosis 2) cancers like lung tumors, especially small cell carcinoma, produce ADH ectopically. Other tumors like cancers of pancreas, duodenum, head and neck may also produce $\mathrm{ADH}$ occasionally 3) lung disorders like pneumonia , bronchial asthma, atelectasis, acute respiratory failure and pneumothorax 4) Hormone administration like vasopressin, desmopressin or oxytocin can cause SIADH by increasing the activity of vasopressor-2 (V2) vasopressin receptor 5) surgical procedures, which includes abdominal and thoracic surgeries and pituitary surgery is also associated with SIADH 6) Both HIV infection and acquired immune deficiency syndrome (AIDS) are associated with SIADH 7) Drugs like oxcarbamazepine, chlorpropamide, cyclophosphamide, sodium valproate, vincristine, ciprofloxacin, opiate, non steroidal anti-inflammatory drugs etc can cause SIADH. ${ }^{14}$

There is urgent need to improve awareness of hyponatremia associated with escitalopram along with its complications to the psychiatrists. It has also been found that in approximately two-thirds of cases rechallenge with the same SSRI resulted in recurrence of hyponatremia, so rechallange should be avoided. ${ }^{15}$

Oxcarbazepine, a keto-analog of carbamazepine. Oxcarbamazepine is used as an antiepileptic drug and some psychiatric conditions. Alone and in combination, oxcarbazepine is a valuable drug. However, it is associated with an increased risk of developing hyponatremia as compared to carbamazepine. ${ }^{16}$

Oxcarbazepine induced hyponatremia is mostly asymptomatic, but its frequency is highly variable and has been estimated at $23-73.3 \%$. Severe or symptomatic hyponatremia is often associated with seizures, neurological damage, mental problems, brain stem herniation and death. ${ }^{17}$ Oxcarbazepine induced hyponatremia occurs in a dose-dependent manner. ${ }^{16}$ When patients treated with oxcarbazepine show seizure aggravation, abnormal behavior, or altered mentality without definite provoking factors, Old age and the use of other drugs for medical conditions increase the risk of hyponatremia, especially aspirin, NSAIDs, CCBs, TCAs, and diuretics.

In this study, difficulty in walking, drowsiness, slurred speech, problem in gait and generalized weakness were the symptoms which are similar to the study done in Korea. ${ }^{18}$ Some antipsychotic drug like trifluoperazine, haloperidol can cause hyponatremia. ${ }^{19}$ As our case series, case 2 received combination of both drugs along with quatiapin which can aggravates hyponatremia.

In this case series, case-4-olanzapine was also prescribed. Atypical antipsychotic (olanzapine) is frequently prescribed to the patients of psychosis. On literature search, olanzapine Is a known drug to cause hyponatremia. Four studies and 91 publications by Meulendijks et al, revealed various case report and case series of hyponatremia due to antipsychotic drugs like olanzapine. ${ }^{20}$ All D2 receptor blockers can cause hyponatremia. The mechanism of hyponatremia may be due to the inhibitory effect of dopamine on release of anti-diuretic hormone $(\mathrm{ADH})$ is blocked by $\mathrm{D} 2$ receptor antagonism. ${ }^{21}$

These cases showed occurrence of hyponatremia treated by various drugs simultaneously. Monitoring of sodium level should be done at regular interval to prevent such type of serious hyponatremia. When these drugs are prescribed concurrently, prescribed should aware of the adverse effects of the drugs.

Funding: No funding sources

Conflict of interest: None declared

Ethical approval: Not required

\section{REFERENCES}

1. Adrogue HJ, Madias NE. Hyponatremia. N Engl J Med. 2000;(342):1581-9.

2. Boscoe A, Paramore C, Verbalis JG. Cost of illness of hyponatremia in the United States. Cost Eff Resour Alloc. 2006;4:10-3.

3. Kumar S, Berl T. Sodium. Lancet. 1998;(352):220-8.

4. Roxanas M, Hibbert E, Field M. Venlafaxine hyponatraemia: incidence, mechanism and management. Aust N Z J Psychiatry. 2007;(41):411-8.

5. Cosgray RE, Hanna V, Davidhizar RE, Smith J. The water-intoxicated patient. Arch Psychiatr Nurs. 1990;(4):308-12.

6. Kirby D, Harrigan S, Ames D. Hyponatraemia in elderly psychiatric patients treated with selective serotonin reuptake inhibitors and venlafaxine: a retrospective controlled study in an inpatient unit. Int J Geriatr Psychiatry. 2002;(17):231-7.

7. Bogunovic O, Sotelo J, Madhusoodanan S. Hyponatremia secondary to antidepressants. Psychiatric Annals. 2003;(33):333-9. 
8. Letmaier M, Painold A, Holl AK, Vergin H, Engel R, Konstantinidis A, et al. Hyponatraemia during psychopharmacological treatment: results of a drug surveillance programme. Int J Neuropsychopharmacol. 2012;(15):739-48.

9. Turnheim K. When drug therapy gets old: pharmacokinetics and pharmacodynamics in the elderly. Exp Gerontol. 2003;(38):843-53.

10. Lin $\mathrm{CH}$, Lu CH, Wang FJ, Tsai MH, Chang WN, Tsai NW, et al. Risk factors of oxcarbazepine-induced hyponatremia in patients with epilepsy. Clin Neuropharmacol. 2010;(33):293-6.

11. Grover S, Biswas P, Bhateja G, Kulhara P. Escitalopram-associated hyponatremia. Psychiatry and Clinical Neurosciences. 2007 Feb 1;61(1):132-3.

12. Nahshoni E, Weizman A, Shefat D, Pik N. A case of hyponatremia associated with escitalopram. J Clin Psychiatry. 2004;(65):17-22.

13. DiPiro JT, Talbert RL, Yee GC, Matzke GR, Wells BG, Posey LM, eds. Pharmacotherapy: a pathophysiologic approach. $5^{\text {th }}$ Ed. New York: McGraw Hill; 2002.

14. Pillai BP, Unnikrishnan AG, Pavithran PV. Syndrome of inappropriate antidiuretic hormone secretion: Revisiting a classical endocrine disorder Indian J Endocrinol Metabolism. 2011;(23):208-15.

15. Liu B, Mittman N, Knowles SR, Shear NH. Hyponatremia and syndrome of inappropriate secretion of antidiuretic hormone associated with the use of selective serotonin reuptake inhibitors: A review of spontaneous reports. Can Med Assoc J. 1996;(155):519-27.

16. Pendlebury SC, Moses DK, Eadie MJ. Hyponatraemia during Oxcarbazepine therapy. Hum Toxicol. 1989;(8):337-44.

17. Adrogue' HJ, Madias NE. Hyponatremia. N Engl J Med. 2000;(342):1581-9.

18. Kim YS, Kim DW, Jung KH, Lee ST, Kang BS, Byun JI, et al. Frequency of and risk factors for oxcarbazepine-induced severe and symptomatic hyponatremia. Seizure-European J of Epile. 2014 Mar 1;23(3):208-12.

19. Peck V, Shenkman L. Haloperidol-induced syndrome of inappropriate secretion of antidiuretic hormone. Clin Pharmacol Ther. 2012;(26):442-4.

20. Meulendijks D, Mannesse CK, Jansen PA, van Marum RJ, Egberts TC. Antipsychotic-induced hyponatraemia: A systematic review of the published evidence. Drug Saf. 2010;(33):101-14.

21. Yamaguchi K, Hama H, Adachi C. Inhibitory role of periventricular dopaminergic mechanisms in hemorrhage induced vasopressin secretion in conscious rats. Brain Res. 1990;(513):335-8.

Cite this article as: Joshi HM, Gupta S, Malhotra S, Patel P. A case series on drug induced hyponatremia: uncommon adverse effect of commonly used drugs. Int J Basic Clin Pharmacol 2018;7:1200-4. 